\title{
A NOTE ON RINGS OF CONTINUOUS FUNCTIONS
}

\author{
J.S. YANG \\ Department of Mathematics and Computer Science \\ University of South Carolina \\ Columbia, South Carolina 29208
}

(Received January 9, 1978, and in revised form March 7, 1978)

ABSTRACT. For a topological space $\mathrm{X}$, and a topological ring $\mathrm{A}$, let $\mathrm{C}(\mathrm{X}, \mathrm{A})$ be the ring of all continuous functions from $\mathrm{X}$ into $\mathrm{A}$ under the pointwise multiplication. We show that the theorem "there is a completely regular space $\mathrm{Y}$ associated with a given topological space $\mathrm{X}$ such that $\mathrm{C}(\mathrm{Y}, \mathrm{R})$ is isomorphic to $C(X, R) "$ may be extended to a fairly large class of topological rings, and that, in the study of algebraic structure of the ring $C(X, A)$, it is sufficient to study $C(X, R)$ if $A$ is path connected.

For a topological space $\mathrm{X}$ and a topological ring $\mathrm{A}$, let $\mathrm{C}(\mathrm{X}, \mathrm{A})$ denote the ring of all continuous functions from $\mathrm{X}$ into $\mathrm{A}$ under the pointwise multiplication. If $A$ is the ring of real numbers $R$ with the usual topology, $\mathrm{C}(\mathrm{X}, \mathrm{R})$ will simply be denoted by $\mathrm{C}(\mathrm{X})$. In [2], the structure of the ring $C(X, A)$, where $X$ is totally disconnected, is studied. The topologies for 
$\mathrm{C}(\mathrm{X}, \mathrm{A})$ considered there are the topology of pointwise convergence, the compact-open topology, and the topology of uniform convergence. Under each of these topologies $\mathrm{C}(\mathrm{X}, \mathrm{A})$ is a topological ring.

In the study of rings of real-valued continuous functions on a topological space, it is usually assumed that $\mathrm{X}$ is completely regular. This assumption of complete regularity on $X$ has no loss of generality as it can be seen in the following.

THEOREM 1. For every topological space $X$, there exists a completely regular space $\mathrm{Y}$ such that $\mathrm{C}(\mathrm{Y})$ is (algebraically) isomorphic to $\mathrm{C}(\mathrm{X})$.

The purpose of this note is to show that the above theorem may be extended to fairly large class of topological rings $A$, and that, in the study of algebraic structure of the ring $C(X, A)$, it is sufficient to study $C(X)$ if $A$ is path connected. All topological spaces considered here are assumed to be Hausdorff.

The following definition is a modification of the one given in [4]. DEFINITION A pair (X,A) of a topological space $\mathrm{X}$ and a topological ring $A$ is called an S-pair, if for each closed subset $C$ of $X$ and $x \notin C$, there exists $f \in C(X, A)$ such that $f(x) \neq 0$ and $Z(f)=\{x \mid f(x)=0\}>C$, where 0 is the zero element of the ring $A$.

It is easy to see that if $\mathrm{X}$ is completely regular and $\mathrm{A}$ is path connected, or if $\mathrm{X}$ is 0-dimensional and $\mathrm{A}$ is any topological ring, then (X,A) is an S-pair.

REMARK If $\left\{\left(\mathrm{X}_{\alpha}, \mathrm{A}_{\alpha}\right): \alpha \in \mathrm{I}\right\}$ is a family of s-pairs, then $\left(\prod_{\alpha \in \mathrm{I}} \mathrm{X}, \prod_{\epsilon} \mathrm{I} \mathrm{A}_{\alpha}\right)$ is also an S-pair, where $\prod_{\alpha \in I} X_{\alpha}$ denotes the product space of the space $X$ while $\prod_{\alpha \in I} \mathrm{~A}_{\alpha}$ denoted the direct product of the rings $\mathrm{A}_{\alpha}$.

PROOF: Let $C$ be a closed subset of $x=\prod_{\alpha \in I} x_{\alpha}$, and let $x \notin C$. Then there exists some basic neighborhood of $\mathrm{x}$ 


$$
\Pi_{\alpha_{1}}^{-1}\left(U_{1}\right) \cap \Pi_{\alpha_{2}}^{-1}\left(U_{2}\right) \cap \ldots \cap \Pi_{\alpha_{n}}^{-1}\left(U_{n}\right)
$$

which is disjoint from $C$, where each $U_{i}$ is open in $x_{\alpha_{i}}, i=1,2, \ldots, n$. For each $i, i=1,2, \ldots, n$, let $f_{i} \in C\left(x_{\alpha_{i}}, A_{\alpha_{i}}\right)$ such that $z\left(f_{i}\right) \supset x_{\alpha_{i}}-U_{i}$, and $f\left(x_{\alpha_{i}}\right) \neq 0_{\alpha_{1}}$, where $x=\left(x_{\alpha}\right)$. Define $g: x \rightarrow A=\prod_{\alpha \in I} A_{\alpha}$ as follows: For $y=\left(y_{\alpha}\right) \in x$, let $g(y)=\left(t_{\alpha}\right)$ where $t_{\alpha}=f_{i}\left(y_{\alpha_{i}}\right)$ if $\alpha=\alpha_{i}$ and $t_{\alpha}=0_{\alpha}$ if $\alpha \neq \alpha_{i}$ for $i=1,2, \ldots, n$. Then $g \in C(X, A), z(g)>C$, and $g(x) \neq 0$.

A topological space $X$ is called a V-space, [3], if for points $p, q, x$, and $y$ of $x$, where $p \neq q$, there exists a continuous functions $f$ of $x$ into itself such that $f(p)=x$ and $f(q)=y$. It is shown [3] that every completely regular path connected space and every zero-dimensional space is a V-space. It is easy to see that if $\left(\mathrm{x}_{\alpha}, \mathrm{A}\right)$ is an S-pair for each $\alpha \in I$, then $\left(\Pi \mathrm{X}_{\alpha \in I}, \mathrm{~A}\right)$ is also an S-pair if the underlying space of $A$ is a V-space. One may ask the question that if $A$ is a topological ring such that $(A, A)$ is an S-pair, is $A$ a V-space? The answer to this question is negative as the following example shows.

EXAMPLE 1. Let $R_{1}$ be the ring of real numbers with the usual topology, and let $R_{2}$ be the ring of integers with the discrete topology. Then $R_{1}$ is path connected while $R_{2}$ is zero-dimensional, thus $\left(R_{1}, R_{1}\right)$ and $\left(R_{2}, R_{2}\right)$ are S-pairs. Hence $\left(R_{1} \times R_{2}, R_{1} \times R_{2}\right)$ is also an S-pair by the remark above. Since $R_{1} \times R_{2}$ is not connected with all components homeomorphic to $R_{1}$, it follows from Theorem 3.5 of [3] that $R_{1} \times R_{2}$ is not a V-space.

Now let $\mathrm{X}$ be a topological space, and $\mathrm{A}$ be a topological ring. For $\mathrm{x}$ and $y$ in $x$, define $x \equiv{ }_{A} y$ if and only if $f(x)=f(y)$ for each $f \in C(X, A)$. Then " $\equiv_{A} "$ is an equivalence relation in $X$. Let $Y_{A}$ be the set of all equivalence classes, and let $T: X \rightarrow Y_{A}$ be the natural map. For each $f \in C(X, A)$, let $f_{T}: Y_{A} \rightarrow A$ be defined by $f_{T}([x])=f(x)$. Then $f_{T}$ is well- 
defined, and $f_{T} \circ T=f$ for each $f \in C(X, A)$.

$$
\text { Let } \begin{aligned}
C_{A} & =\left\{f_{T} \in A^{Y} \mid f \in C(X, A)\right\} \\
& =\left\{g \in A^{Y} \mid g \circ T \in C(X, A)\right\}
\end{aligned}
$$

and let $\tau_{A}$ be the weak topology on $\mathrm{Y}_{\mathrm{A}}$ induced by the family $\mathrm{C}_{\mathrm{A}}$. Note that the construction of the space $Y_{A}$ is analogous to that of the space $Y$ of Theorem 1 .

THEOREM 2 (1) The topological space $\left(Y_{A}, \tau_{A}\right)$ is Hausdorff.

(2) $\left(Y_{A}, \tau_{A}\right)$ is completely regular.

(3) The mapping $\mathrm{T}: \mathrm{X} \rightarrow\left(\mathrm{Y}_{\mathrm{A}}, \tau_{\mathrm{A}}\right)$ is continuous.

(4) The mapping $\phi: g \rightarrow g \circ T$ of $C\left(Y_{A}, A\right)$ onto $C(X, A)$ is a continuous isomorphism, where $C(Z, A)$ is assumed to have the compact-open topology.

PROOF: (3) and (4) are clear.

To show (1), let $y_{1}, y_{2} \in Y_{A}$, where $y_{1}=\left[x_{1}\right], y_{2}=\left[x_{2}\right]$, and $y_{1} \neq y_{2}$. Then there exists $f \in C(X, A)$ such that $f\left(x_{1}\right) \neq f\left(x_{2}\right)$. Thus $f_{T}\left(y_{1}\right) \neq f_{T}\left(y_{2}\right)$. If $V_{1}$ and $V_{2}$ are open sets in $A$ such that $f_{T}\left(y_{1}\right) \in V_{1}$ for $i=1,2$, and $\mathrm{V}_{1} \cap \mathrm{V}_{2}=\phi$, then $\mathrm{f}_{\mathrm{T}}^{-1}\left(\mathrm{~V}_{1}\right) \cap \mathrm{f}_{\mathrm{T}}^{-1}\left(\mathrm{~V}_{2}\right)=\phi$. Hence $\left(\mathrm{Y}_{\mathrm{A}}, \tau_{\mathrm{A}}\right)$ is Hausdorff. For (2), let $x \in U=f_{T_{1}}^{-1}\left(V_{1}\right) \cap f_{T_{2}}^{-1}\left(V_{2}\right) \cap \ldots \cap f_{T_{n}}^{-1}\left(V_{n}\right)$, where each $V_{i}$ is open in $A$, and $f_{T_{i}} \in C_{A}$, $i=1,2, \ldots, n$. Then $f_{T_{i}}(x) \in v_{i}$ for each $i=1,2, \ldots, n$. For each $i$, there exists $g_{i} \in C(A,[0,1])$ such that $g_{i}\left(f_{T_{i}}(x)\right) \neq 0$ and $g_{i}\left(A-V_{i}\right)=0$. If we let $h=\left(g_{1} \circ f_{T_{1}}\right)\left(g_{2} \circ f_{T_{2}}\right) \ldots\left(g_{n} \circ f_{T_{n}}\right)$, then $h \in C\left(Y_{A}\right)$, and $h(x) \neq 0$ but $h(y)=0$ for $y \notin U$. Hence $\left(Y_{A}, \tau_{A}\right)$ is completely regular.

It is noted that the map $\mathrm{T}$ need not be a quotient map as the following example, [1], shows.

EXAMPLE 2. Let $S$ denote the subspace of $R^{2}$ obtained by deleting $(0,0)$ and all points $\left(\frac{1}{n}, y\right)$ with $y \neq 0$ and $n \in N$. Define $\pi(x, y)=x$ for all $(x, y) \in s$. 
Let $\mathrm{X}$ be the quotient space of $\mathrm{S}$ induced by the mapping $\pi$ then $\mathrm{X}$ can be identified as the set of real numbers endowed with the largest topology for which the mapping $\pi$ is continuous. It is demonstrated in [1] that $X$ is Hausdorff, not completely regular, $Y_{R}$ is the space of real numbers, and that the mapping $T$ is not a quotient map.

THEOREM 3 If the ring $A$ is path connected, then

(1) $\left(\left(Y_{A}, \tau_{A}\right), A\right)$ is an S-pair

(2) $\mathrm{Y}_{\mathrm{A}}=\mathrm{Y}_{\mathrm{R}}$

(3) $\tau_{A}=\tau_{R}$.

PROOF: Since $A$ is assumed to be path connected while $\left(Y_{A}, \tau_{A}\right)$ is completely regular by Theorem 2, (1) is clear.

To show (2) it is sufficient to show that $x \equiv_{R} y$ if and only if $x \equiv{ }_{A} y$ whenever $\mathrm{x}, \mathrm{y} \in \mathrm{X}$. Let $\mathrm{x} \equiv_{\mathrm{R}^{\mathrm{y}}} \mathrm{y}$ but suppose that $\mathrm{x} \neq_{\mathrm{A}} \mathrm{y}$. Then there exists $f \in C(X, A)$ such that $f(x) \neq f(y)$. Let $g \in C(A)$ such that $g \circ f(x) \neq g \circ f(y)$. This would imply that $x$ f $_{R} y$ since $g \circ f \in C(X)$, a contradiction. Conversely, if $x \equiv \equiv_{A} y$ but $x \neq{ }_{R} y$. Then there exists $f \in C(X)$ such that $f(x) \neq \dot{f}(y)$. Then there exists $h \in C(R, A)$ such that $h(f(x))=0$ but $h(f(y))=t \neq 0$. If $\mathrm{g}=\mathrm{h} \circ \mathrm{f}$, then $\mathrm{g} \in \mathrm{C}(\mathrm{X}, \mathrm{A})$ but $\mathrm{g}(\mathrm{x}) \neq \mathrm{g}(\mathrm{y})$ which leads to a contradiction again.

Finally we shall prove (3). Since A is completely regular C(A) separates points from closed sets in $A$, thus sets of the form $k^{-1}(V)$, where $k \in C(A)$ and $V$ open in $R$, form a subbase for the topology of $A$. Let $f_{T}^{-1}(U)$ be a subbasic open set in $\tau_{A}$. Then $U$ is open in $A$, hence we may let $U=n_{i=1}^{n} k_{i}^{-1}\left(V_{i}\right)$, where for each $i=1,2,3, \ldots, n, k_{i} \in C(A)$ and $v_{i}$ open in $R$. Thus $f_{T}^{-1}(U)=f_{T}^{-1}\left(n_{i=1}^{n} k_{i}^{-1}\left(V_{i}\right)\right)=\prod_{i=1}^{n} f_{T}^{-1}\left(k_{i}^{-1}\left(V_{i}\right)\right)=n_{i=1}^{n}\left(k_{i} \circ f_{T}\right)^{-1}\left(V_{i}\right)$. Since $k_{i} \circ f_{T} \in R^{Y_{A}}$ and $Y_{A}=Y_{R}$ by (2), this implies that $\tau_{A} \subset \tau_{R}$. Conversely, let 
$h^{-1}(U)$ be a subbasic open set in $\tau_{R}$, where $U$ is open in $R$ and $h \circ T \in C(X)$. Let $y \in h^{-1}(U)$. Since $\left(Y_{R}, \tau_{R}\right)$ is completely regular, $\left(\left(Y_{R}, \tau_{R}\right), A\right)$ is an $S-p a i r$, hence there exists $f \in C\left(Y_{R}, A\right)$ such that $f(y) \neq 0$ but $f\left(Y_{R}-h^{-1}(U)\right)=0$. Then $y \in f^{-1}(A-\{0\}) \subset h^{-1}(U)$. Since $f \circ T \in C(X, A)$ and $f \in A^{Y}$, this shows that $h^{-1}(U) \in \tau_{A}$. Hence $\tau_{R} \subset \tau_{A}$.

The above theorem shows that, within the category of path connected topological rings, the space $Y_{A}$ is independent of the ring $A$.

\section{REFERENCES}

1. L. Gillman and M. Jerrison, Rings of continuous functions, D. Van Nostrand, N. Y. 1960 .

2. I. Kaplansky, Topological rings, Amer. J. Math. 69 (1947), 153-183.

3. K. D. Magill, Jr., Some homomorphism theorems for a class of semigroups, Proc. London Math. Soc. 15 (1965), 517-526.

4. J. S. Yang, Transformation groups of automorphisms of $c(X, G)$, Proc. Amer. Math. Soc. 39 (1973), 619-624.

KEY WORDS AND PHRASES. Continuous functions, completely regular space, topological ring, S-pair, compact-open topology.

AMS (MOS) SUBJECT CLASSIFICATIONS (1970). 54C35, $54 C 40$. 


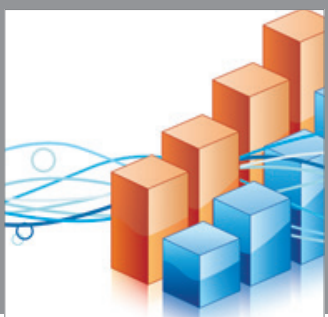

Advances in

Operations Research

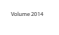

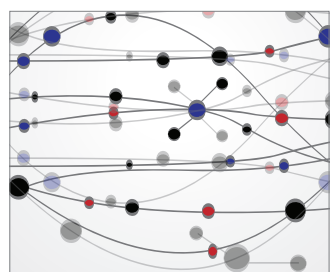

\section{The Scientific} World Journal
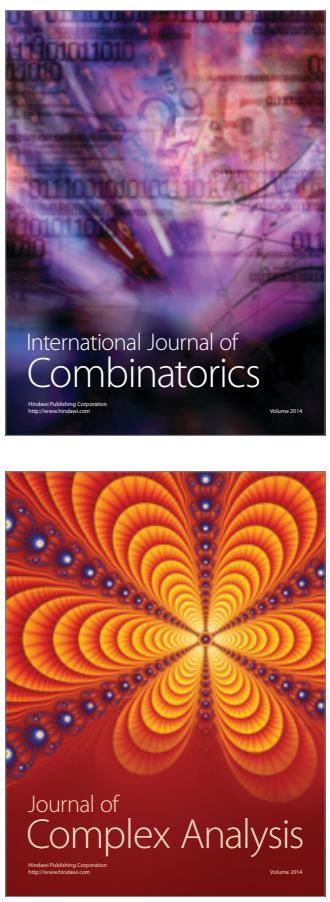

International Journal of

Mathematics and

Mathematical

Sciences
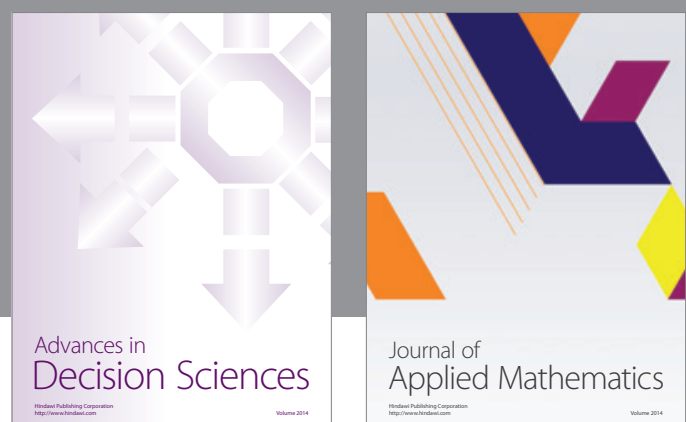

Journal of

Applied Mathematics
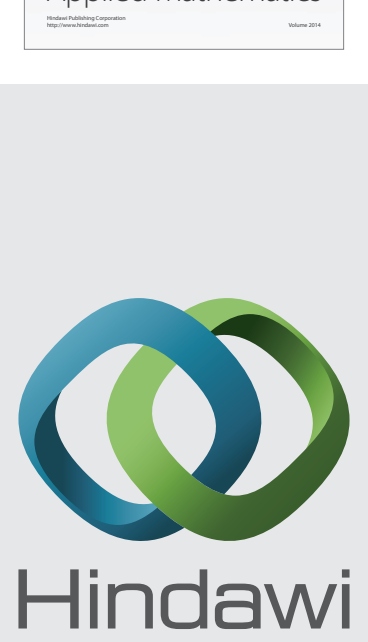

Submit your manuscripts at http://www.hindawi.com
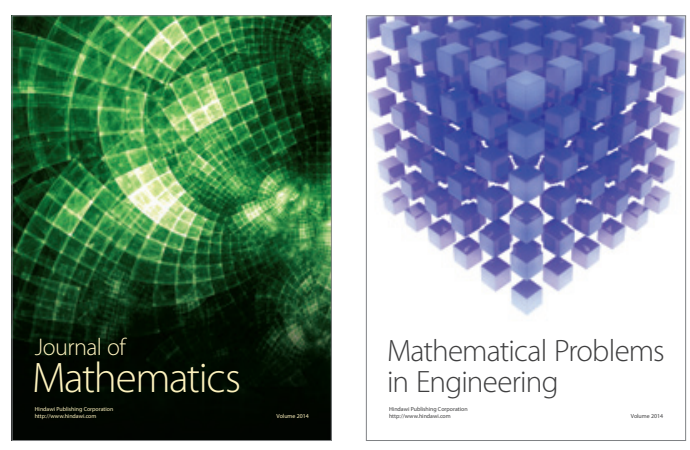

Mathematical Problems in Engineering
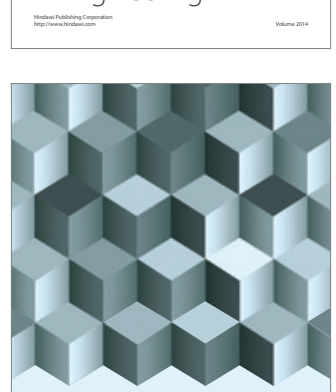

Journal of

Function Spaces
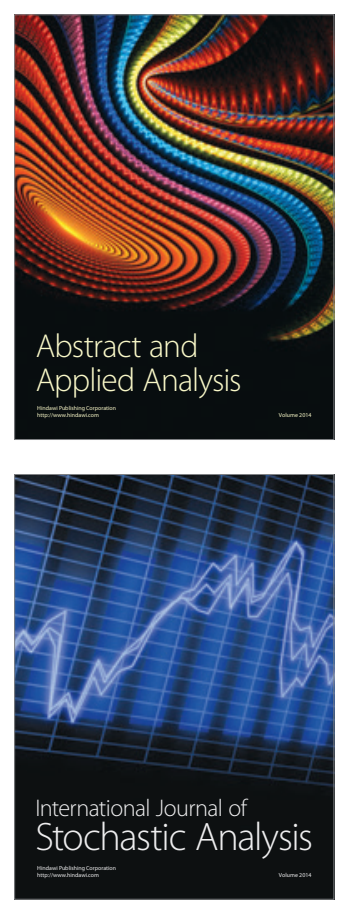

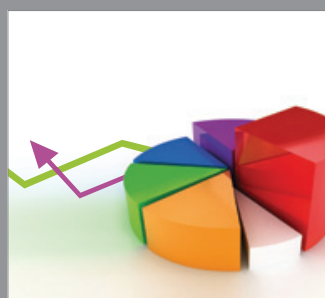

ournal of

Probability and Statistics

Promensencen
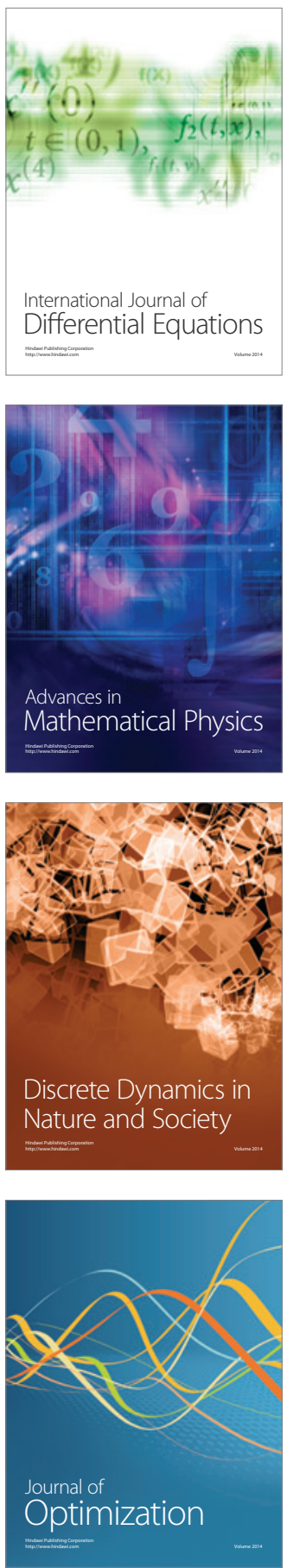